\title{
Escape, capture, and levitation of matter in Eddington outbursts
}

\author{
A. Stahl ${ }^{1}$, W. Kluźniak ${ }^{2}$, M. Wielgus ${ }^{3}$, and M. Abramowicz ${ }^{1,2}$ \\ 1 Physics Department, Gothenburg University, 412-96 Göteborg, Sweden \\ e-mail: gusstaad@student.gu.se, marek.abramowicz@physics.gu.se \\ 2 Nicolaus Copernicus Astronomical Center, ul. Bartycka 18, 00-716 Warszawa, Poland \\ e-mail: wlodek@camk.edu.pl \\ 3 Institute of Micromechanics and Photonics, ul. św A. Boboli 8, 02-525 Warszawa, Poland \\ e-mail: maciek.wielgus@gmail.com
}

Received 28 March 2013 / Accepted 26 May 2013

\begin{abstract}
Context. An impulsive increase in luminosity by one half or more of the Eddington value will lead to ejection of all optically thin plasma from Keplerian orbits around the radiating star, if gravity is Newtonian and the Poynting-Robertson drag is neglected. Radiation drag may bring some particles down to the stellar surface. On the other hand, general relativistic calculations show that gravity may be balanced by a sufficiently intense radiation field at a certain distance from the star.

Aims. We investigate the motion of test particles around highly luminous stars to determine conditions under which plasma may be ejected from the system.

Results. In Einstein's gravity, if the outburst is close to the Eddington luminosity, all test particles orbiting outside an "escape sphere" will be ejected from the system, while all others will be captured from their orbits onto the surface of another sphere, which is well above the stellar surface, and may even be outside the escape sphere, depending on the value of luminosity. Radiation drag will bring all the captured particles to rest on this "Eddington capture sphere", where they will remain suspended in an equilibrium state as long as the local flux of radiation does not change and remains at the effective Eddington value.
\end{abstract}

Key words. stars: neutron - stars: winds, outflows - X-rays: binaries - scattering - accretion, accretion disks

\section{Introduction}

It is widely believed that in luminous compact sources, such as the accreting neutron stars in low-mass X-ray binaries, PoyntingRobertson drag will tend to increase the accretion rate as the luminosity of the source increases. Not intending to disagree that in steady sources radiation drag may enhance the accretion rate, here we wish to point out that a sudden increase in luminosity may in fact have the opposite effect in optically thin plasma: atoms (or ions) subjected to increased radiation pressure will tend to move out, away from the stellar surface, PoyntingRobertson drag notwithstanding.

In Newtonian dynamics, the effects of increased radiation pressure are straightforward to compute. A simple application of orbital mechanics reveals that an increase in luminosity by one half of the Eddington value ${ }^{1}$ is sufficient to eject optically thin plasma from its Keplerian orbits at any initial distance from the star.

In Einstein's general relativity (GR) this remains qualitatively true at a large enough distance from the star - in the optically thin regime a sudden increase in luminosity of sufficient magnitude will unbind the orbiting fluid. However, closer to the star, competing effects of gravity and radiation pressure may lead to very nonintuitive behavior, particularly when combined with the effects of radiation drag. We show in an exact GR calculation that if the luminosity at infinity is close to the Eddington value, optically thin matter in the vicinity of a compact star is neither

\footnotetext{
1 More precisely, an impulsive increase in luminosity by one half of the difference between the most recent steady value and the Eddington luminosity is sufficient to unbind a non-relativistic test particle (Kluźniak 2013).
}

ejected from the system nor accreted onto the star. Instead, it will collapse onto the surface of an imaginary sphere, whose radius is a function of the stellar radius and the luminosity (Eq. (3)). Rather than orbit the star, fluid on this Eddington capture sphere remains at rest in a state of equilibrium (Stahl et al. 2012), suspended above the stellar radius, in what is to all intents and purposes a state of levitation.

\section{The Eddington capture sphere}

In Einstein's general relativity, the redshifted luminosity $L(r)$ of an isotropic star decreases according to

$L(r)=L_{\infty} /\left(1-R_{\mathrm{Schw}} / r\right)$.

The effective Eddington luminosity at $r$, i.e., the luminosity at which radiation pressure balances gravity, is

$L_{\text {eff }}(r)=L_{\text {Edd }}\left(1-R_{\text {Schw }} / r\right)^{-1 / 2}$.

For a given luminosity $L_{\infty}$, a static balance between the radiation pressure force and gravitational pull is then achieved at the radius at which $L(r)=L_{\mathrm{eff}}(r)$ (Phinney 1987). The formula for this unique radius, $r_{\text {Edd }}\left(L_{\infty}\right)$, can be written in the form

$\frac{r_{\text {Edd }}}{R_{\text {Schw }}}=\left[1-\left(\frac{L_{\infty}}{L_{\text {Edd }}}\right)^{2}\right]^{-1}$.

We use standard Schwarzschild coordinates, with the Schwarzschild radius $R_{\text {Schw }}=2 G M / c^{2}$, and $L_{\infty} / L_{\text {Edd }}$ the stellar luminosity at infinity in units of the Eddington value, $L_{\mathrm{Edd}}=4 \pi G M m_{\mathrm{p}} c / \sigma$, where $\sigma$ is the Thomson cross-section. 
Strictly speaking the formula is true for an optically thin shell of hydrogen plasma, but similar formulae hold for other compositions of the fluid, as well as other photon momentum absorption processes. Abramowicz et al. (1990) showed that $r_{\text {Edd }}$ is a position of stable equilibrium for test particles (with proton mass, and Thomson cross-section for photon momentum absorption) moving radially in the combined gravitational and radiation fields of a spherical, non-rotating, isotropically radiating compact star.

It is now known that the sphere at $r_{\text {Edd }}$ is also a position of equilibrium with respect to non-radial perturbations, and that in fact particles from a wide class of trajectories will be captured on this sphere, because of radiation drag (see e.g., Bini et al. 2009; Oh et al. 2011; Stahl et al. 2012, for work in the Schwarzschild metric). Hence, we refer to it as the Eddington capture sphere (ECS). For Kerr metric results, see e.g., Oh et al. (2010); Bini et al. (2011).

\section{Equations of motion}

We carry out all calculations in the Schwarzschild metric using the standard Schwarzschild spherical coordinates, and extend the treatment of Abramowicz et al. (1990) to non-radial motion; we use their stress-energy tensor of radiation $T^{(i)(k)}$, which was calculated in the stationary observer tetrad assuming isotropic emission from the stellar surface. An explicit form of the stressenergy tensor is given in the Appendix, along with a summary of the derivation of the equations of motion. Spherical symmetry of the problem assures that for any set of initial conditions the trajectory of a test particle is confined to a single plane, which we take to be the equatorial plane $\theta=\pi / 2$.

From this point on we take $G=1=c$, and scale all radii with $M$ (i.e., one half of the Schwarzschild radius). The proper time is given by the Schwarzschild metric restricted to the equatorial plane

$\mathrm{d} \tau^{2}=\left(1-\frac{2}{r}\right) \mathrm{d} t^{2}-\left(1-\frac{2}{r}\right)^{-1} \mathrm{~d} r^{2}-r^{2} \mathrm{~d} \phi^{2}$.

We introduce $B=1-2 / r$, and denote the four-velocity by $u^{i}$, so $u^{t}=\mathrm{d} t / \mathrm{d} \tau$. The angular frequency observed at infinity is $\Omega=(\mathrm{d} \phi / \mathrm{d} \tau) / u^{t}$, when discussing circular orbits we use its Keplerian value $\Omega_{K}(r)=1 / r^{3 / 2} \equiv v_{K}(r) / r$. In the figures, we find it convenient to use $v^{\phi} \equiv r \Omega$, scaled with the coordinate speed of light, at those (initial) moments when the radial velocity is zero, and $v^{r} \equiv \mathrm{d} r / \mathrm{d} t$ when the azimuthal velocity is zero. The stellar radius is denoted by $R$.

We make the standard assumption of efficient electromagnetic coupling between protons and electrons and, as is usual in the discussion of Eddington luminosity, take the mass of the test particle to be equal to that of the proton. In so doing, we neglect possible effects of charge separation discussed by Walker (1988). The effects of Poynting-Robertson drag in steady disk accretion were considered by Walker \& Mészáros (1989). We consider a time-dependent problem. On the assumption that the test particle is absorbing momentum (from the radiation field) with the Thomson cross-section in its rest frame, thus suffering a rest-frame force of $(\sigma / c) \times$ flux, the particle trajectory is described by two coupled, second order differential equations:

$$
\begin{aligned}
\frac{\mathrm{d}^{2} r}{\mathrm{~d} \tau^{2}}= & \frac{k}{\pi I(R) R^{2}}\left(B T^{(r)(t)} u^{t}-\left[T^{(r)(r)}+\varepsilon\right] \frac{\mathrm{d} r}{\mathrm{~d} \tau}\right) \\
& +(r-3)\left(\frac{\mathrm{d} \phi}{\mathrm{d} \tau}\right)^{2}-\frac{1}{r^{2}}, \\
\frac{\mathrm{d}^{2} \phi}{\mathrm{d} \tau^{2}}= & -\frac{\mathrm{d} \phi}{\mathrm{d} \tau}\left(\frac{k}{\pi I(R) R^{2}}\left[T^{(\phi)(\phi)}+\varepsilon\right]+\frac{2}{r} \frac{\mathrm{d} r}{\mathrm{~d} \tau}\right),
\end{aligned}
$$

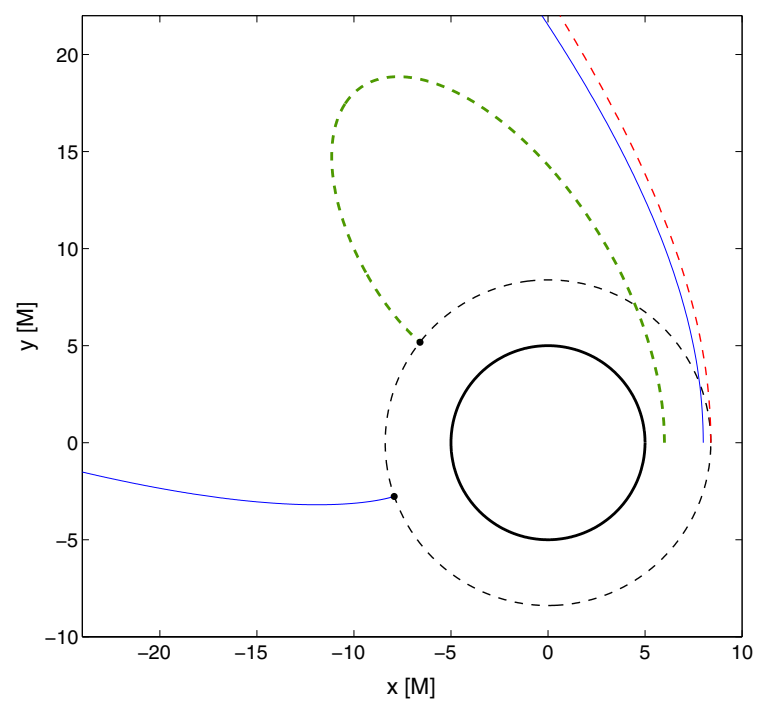

Fig. 1. Trajectories of particles leaving their circular orbits following an impulsive increase in luminosity from zero to that value $(k=1.45$ for $R=5$ ) at which the escape sphere and the Eddington capture sphere coincide. Particles initially in circular orbits at (or outside) the escape sphere leave the system on outward bound trajectories (thin dashed red line). Those from initial orbits inside the escape sphere, turn back and are captured by the ECS, on which they remain at rest in their final position (thick dots).

with

$$
\begin{aligned}
\varepsilon= & B T^{(t)(t)}\left(u^{t}\right)^{2}+B^{-1} T^{(r)(r)}\left(\frac{\mathrm{d} r}{\mathrm{~d} \tau}\right)^{2} \\
& +r^{2} T^{(\phi)(\phi)}\left(\frac{\mathrm{d} \phi}{\mathrm{d} \tau}\right)^{2}-2 T^{(r)(t)} u^{t} \frac{\mathrm{d} r}{\mathrm{~d} \tau} .
\end{aligned}
$$

The parameter $k$ is the stellar surface luminosity in Eddington units,

$k=\frac{L(R)}{L_{\mathrm{Edd}}}=4 \pi^{2} R^{2} \frac{I(R)}{L_{\mathrm{Edd}}}=\frac{L_{\infty}}{L_{\mathrm{Edd}}}\left(1-\frac{2}{R}\right)^{-1}$.

Because $B T^{(r)(t)}=\pi I(R)(1-2 / R)\left(R^{2} / r^{2}\right)$, Eqs. (5), (6) allow a static solution $r(\tau)=r_{\text {Edd }}($ Eq. $(3))$, provided that

$(1-2 / R)^{1 / 2} \leq L_{\infty} / L_{\text {Edd }}<1$.

By integrating Eqs. (5) and (6), we are able to find the particle orbits, such as the ones illustrated in Fig. 1

All numerical results presented below were obtained with the Dormand-Prince method, which is a fourth-order accuracy, adaptive Runge-Kutta type integrator.

\section{Escape from the vicinity of a luminous star}

In the absence of radiation, it is easy enough to determine whether or not a test particle will leave the system. A particle is bound or unbound depending on its angular momentum and energy. An unbound test particle is certain to escape to infinity, if only it is outward going, i.e., if $u^{r}(0) \geq 0$, while incoming particles may, or may not, be captured by the star depending on their impact parameter.

The presence of radiation drag complicates matters, as energy and angular momentum are no longer constants of the motion. One needs to compute the actual trajectory for given initial 


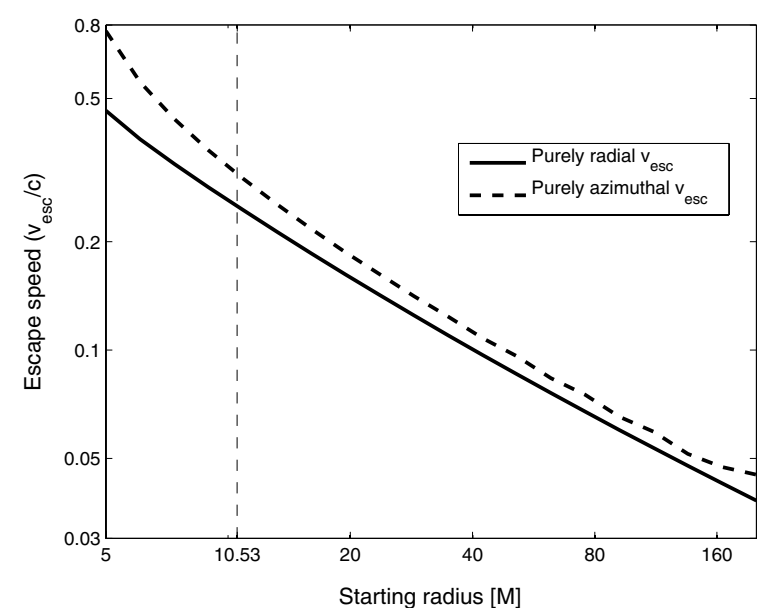

Fig. 2. Escape speeds normalized to the speed of light, as a function of the starting radius, for $R=5, L_{\infty}=0.9 L_{\mathrm{Edd}}$. Solid line corresponds to radial motion. The Eddington capture sphere is at the position marked with a vertical dashed line.

conditions to find out whether or not the particle leaves the system. In this paper we only consider trajectories with $u^{r}(0) \geq 0$. For examples of inward bound trajectories see, e.g., Stahl et al. (2012).

We have integrated the equations of motion to find trajectories that are not bounded ${ }^{2}$ and found the minimum ("escape") velocities required for escape from the system. These depend on the initial velocity vector, with the highest value of the escape speed corresponding to initial velocities tangent to a circle concentric with the spherical star ("purely azimuthal" initial motion), and the lowest for radial motion $\left(v^{\phi}=0\right)$. These two limiting cases are shown in Fig. 2 as a function of the radius when the stellar radius and luminosity are $R=5$, and $L_{\infty}=0.9 L_{\text {Edd }}$ or $k=1.5$.

Technically, the criterion we used to decide whether or not a test particle escapes to infinity was to integrate the equations of motion until the particle turned around $\left(u^{r}<0\right)$, or conversely, reached $r=1500 R_{\mathrm{Schw}}$. In the latter case, motion was always radial to a high accuracy, and radiation drag negligible. The particle was deemed to be on a bounded trajectory if its (relativistic) specific energy at $r=1500 R_{\text {Schw }}$ was less than unity. If the specific energy was $\geq 1$, the particle was classified as escaping the system.

Except at the very highest luminosities, it is rather hard for particles to escape from the ECS. Figure 3 shows the values of escape velocities from the ECS, as a function of the luminosity parameter $k$ of Eq. (8).

In the limit of $L_{\infty} \rightarrow L_{\text {Edd }}$, the ECS becomes infinitely large, $r_{\text {Edd }} \rightarrow \infty$, and the escape velocity goes to zero. In the opposite (lower) limit of Eq. (9), $r_{\text {Edd }}=R$. In this case, the escape speed from the stellar surface, which now coincides with the ECS, exceeds one half of the speed of light (Fig. 4) for all $R \leq 5$. This is not an unusually high value, if one recalls (Kluźniak \& Wagoner 1985 ) that in the Schwarzschild metric the highest Keplerian orbital speed is $c / 2$ (in the marginally stable orbit, a.k.a. the ISCO, at $r=6$ ), however, at first sight it may seem surprising that the escape speed remains so high even in the presence of the effective Eddington luminosity of radiation, whose pressure balances gravity, Eq. (2). As a reminder we note that in this context the escape speed has a somewhat different meaning than usually.

\footnotetext{
2 Trajectories that are not bounded cannot be enclosed in any sphere, however large.
}

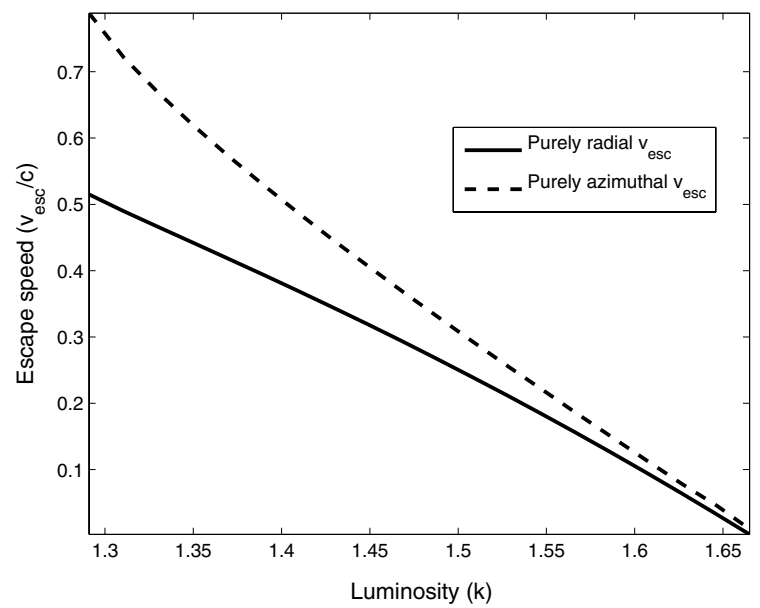

Fig. 3. Escape speeds (normalized to the speed of light) from the Eddington capture sphere vs. luminosity for $R=5$. The whole range of luminosities is shown, for which there is an ECS.

It is not the speed necessary for the particle to be unbound, i.e., its energy to be larger than its rest mass. Rather, it is that speed which is sufficient to "overcome" radiation drag, so that the orbit is not bounded, i.e., a speed sufficient for the particle to escape to infinity in spite of continual loss of angular momentum to the radiation field.

\section{Impulsive change in luminosity}

The question we are addressing in this paper is whether or not an optically thin corona will be ejected from a system undergoing an impulsive increase in luminosity to the effective Eddington value, and what becomes of the particles which are not ejected. Astrophysically, such a situation may be encountered, e.g., in X-ray bursters (see Sect. 6). In Newtonian mechanics and gravity, this question was answered by Kluźniak (2013), who found that in the absence of radiation drag all particles will be ejected if the luminosity increases above a critical value, equal to $\left(L+L_{\mathrm{Edd}}\right) / 2$, where $L$ was the luminosity prior to the increase. To solve the problem in full GR with radiation drag included, we integrate Eqs. (5) and (6).

Consider the trajectory of a test particle in a circular orbit at $r$ about a star whose luminosity will undergo an impulsive change from zero to $L(R)$. Thus, the initial conditions correspond to a particle with $v^{r}=0$, and $v^{\phi}=v_{K}$ appropriate for that circular orbit, $v_{K}$ being the Keplerian speed defined following Eq. (4). We begin the integration at that moment in which the luminosity at $r$ jumps from zero to $L(r)$ of Eq. (1), and find that this initial $v^{\phi}$ is higher than the azimuthal escape speed for all $r$ greater than some critical value, which is a function of $R$ and $L(R)$, while it is lower for all radii below that critical value. Accordingly, we introduce the escape sphere dividing space into an outer region, from which all test particles will be hurled out to infinity by the impulsive change in luminosity, and the inner region, between the stellar surface and the escape sphere, from which the particles do not escape the system (cf. Fig. 5).

The fate of particles within the escape sphere is rather interesting. Because of radiation drag, for luminosities below the lower limit of Eq. (9), the particles would have ended up on the stellar surface. However, for the luminosities considered here they all come to rest on the ECS. This is illustrated in Fig. 1, which shows an escaping trajectory of a particle previously in 


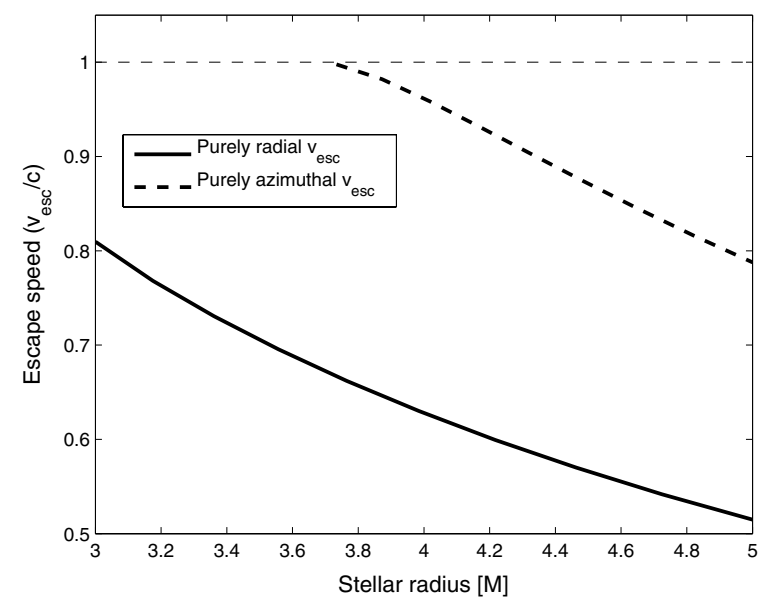

Fig. 4. Escape speeds from the Eddington capture sphere when it coincides with the stellar surface, as a function of the stellar radius, for purely radial and azimuthal initial velocities.

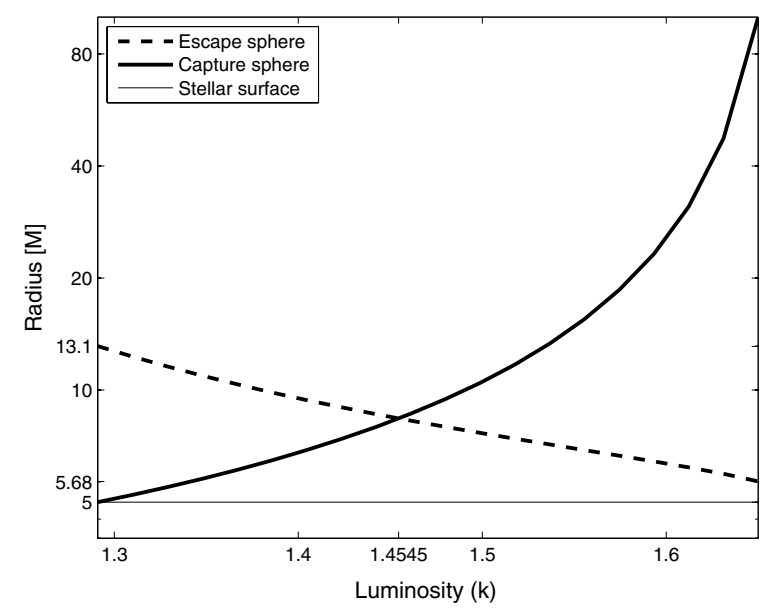

Fig. 5. Radii of the escape sphere and the Eddington capture sphere as a function of the stellar luminosity $(R=5)$. The two spheres coincide at the critical value of $k=1.45$, or $L_{\infty}=0.873 L_{\mathrm{Edd}}$, corresponding to $r_{\text {Edd }}=8.40$.

circular orbit on what is now the escape sphere, as well as bounded trajectories from circular orbits of lower radii.

As it turns out, the escape sphere may be inside or outside the ECS. Thus, for a high enough luminosity, all particles initially outside the escape sphere escape to infinity, while those inside it migrate outside to settle on the ECS. Figure 5 shows the radii of the escape sphere and of the capture sphere, as a function of the luminosity parameter. The two coincide for a critical value of the luminosity parameter, which we find to be $k_{\text {crit }}=1.45$ for $R=5$, the orbits shown in Fig. 1 correspond to this case.

For particles that are initially well within the escape sphere, the migration time to the ECS is rather short. This is illustrated in Fig. 6. For a $2 M_{\odot}$ star the unit of time is $2 G M_{\odot} / c^{3} \approx 10^{-5} \mathrm{~s}$. Thus, all times shown in the figure correspond to a fraction of a second, and this is how long the high luminosity has to be sustained for the particles to collect on the ECS.

\section{Discussion}

While our computations were performed for test particles, the main results should also hold for plasma, in which internal dissipation of energy may occur. Clearly, the particles that eventually

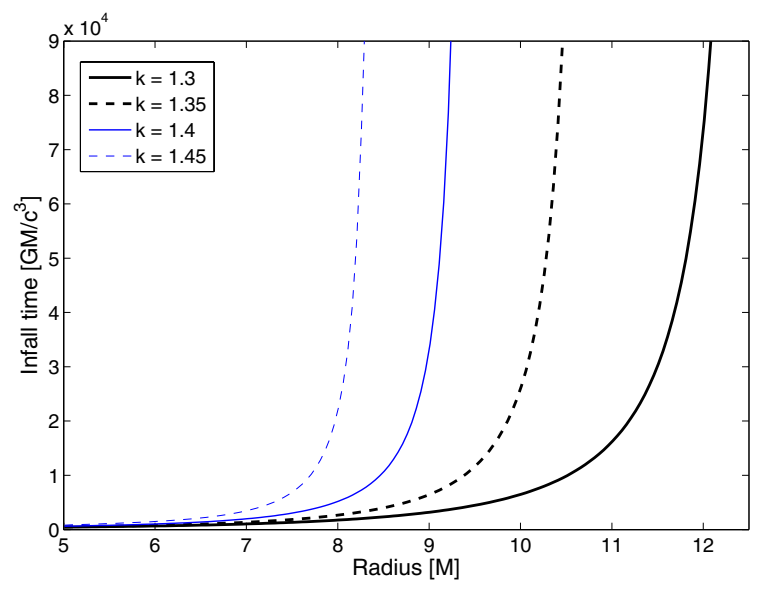

Fig. 6. Infall time onto the Eddington capture sphere from various initial radii within the escape sphere. Note that the tick marks on the vertical scale are separated by $10^{4} \mathrm{GM} / \mathrm{c}^{3}$.

settle on the ECS lose all their kinetic energy (and angular momentum), and it makes little difference whether some of it is first redistributed to other particles.

As the radiation front of increased flux travels at the speed of light, all plasma particles are accelerated and the outbound particles do not run into other particles departing from larger radii. The particles that are on unbounded trajectories very quickly approach radial motion, because of radiation drag. Very little plasma compression and dissipation is likely to occur in this situation. In any case, the corona is assumed to be radiatively inefficient, so any dissipation of energy does not lead to binding plasma that is unbound - the total energy of the plasma remains positive. However, there are two serious limitations of the current work, which should be overcome in future calculations. We have assumed spherical symmetry, while the most luminous, and interesting, astronomical sources accrete in a disk-like geometry. Further, in the microscopic domain we have neglected the transfer of momentum between the scattered (or re-emitted) photons and the test particle - only the momentum transferred by the absorbed photons was included in the calculation. Thus the radius of the escape sphere is likely to have been underestimated. On the other hand, the position of the ECS is insensitive to this assumption, particles at rest on the ECS radiate isotropically, and the only momentum transfer is from the absorption process included in our calculation.

The future will tell whether the theoretical finding presented here have any application to X-ray bursts, which are spherically symmetric to a high degree, and how they affect the estimates of neutron star radius, as in, e.g., Damen et al. (1990). As the precise value of the stellar radius and mass is not known, it is hard at present to translate the observed flux from the neutron star to a precise value of the luminosity $k$ parameter. Should a direct observational determination of $k$ turn out to be possible, a value for the stellar radius would immediately follow (cf. Eq. (8)). However, we note that X-ray bursters are thought to attain Eddington luminosity, e.g., Lewin et al. (1993), and a recent burst in the neutron star 2S 0918-549 has exhibited an interesting achromatic oscillation, which has been interpreted as an accretion disk response to super-Eddington fluxes in an X-ray burst (in 't Zand et al. 2011). Wielgus et al. (2012) point out that, in principle, a luminosity oscillation may be related to the appearance and disappearance of the ECS. Very interesting luminosity variations were also reported in the $\mathrm{Z}$ sources, or rather, in the $\mathrm{Z}$ phase of bright LMXBs (Lin et al. 2009), and in some 
microquasars - of course, these are precisely the sources where spherical symmetry is broken, and more work is needed before any reliable conclusions can be reached on the relevance to these sources of the phenomena discussed here.

Perhaps it is worth noting that super-Eddington luminosity may also be present among some accreting black holes, notably ULXs (Feng \& Soria 2011; Gladstone et al. 2011), but also in supermassive black holes, (e.g., Kollmeier et al. 2011; Nobuta et al. 2012), and microquasars, such as V4641 Sgr in outburst (Revnivtsev et al. 2002).

\section{Conclusions}

We have shown in a GR calculation including radiation drag that a sudden change in luminosity from zero to about the (effective) Eddington value leads to ejection of all test particles outside a certain sphere (the escape sphere). In Newtonian physics all particles outside the stellar surface are either ejected, or not, depending on the luminosity jump (Kluźniak 2013), when radiation drag is neglected. In Einstein's gravity, the redshifted luminosity drops with radius, and this effect, combined with radiation drag, introduces a radius dependence to the problem, thus allowing some particle to be left behind in the system. At the high final luminosities considered here, the non-escaping particles are not accreted by the star. Instead, they lose all kinetic energy and angular momentum and migrate to the ECS, typically within a fraction of a second, where they remain at rest as long as the local flux of radiation corresponds to the effective Eddington value.

We expect our work to be applicable to the optically thin coronae of variable X-ray sources. Future work will show how the position of the escape sphere depends on the initial luminosity of the system (Mishra et al. 2013).

Acknowledgements. We thank Saul Rappaport and Wenfei Yu for inspiring discussions. In addition, Saul Rappaport's help was invaluable in validating our codes. Research supported in part by Polish NCN grants UMO2011/01/B/ST9/05439, and N N203 511238, and the Czech grant MSM 4781305903, as well as by a Swedish VR grant.

\section{Appendix A}

Here, we present an abbreviated derivation of Eqs. (5), (6). The acceleration of the particle is in general given by

$a^{i}=u^{k} \nabla_{k} u^{i}=\frac{\mathrm{d}^{2} x^{i}}{\mathrm{~d} s^{2}}+\Gamma_{j k}^{i} u^{j} u^{k}=\frac{1}{M} \frac{\mathrm{d}}{\mathrm{d} \tau}\left(u^{i}\right)+\Gamma_{j k}^{i} u^{j} u^{k}$,

where $\mathrm{d} s^{2}=M^{2} \mathrm{~d} \tau^{2}$. Taking the force of radiation to be

$c^{2} m a^{i}=\frac{\sigma}{c} F^{i}$,

we obtain the equation of motion

$\frac{\mathrm{d}}{\mathrm{d} \tau}\left(u^{i}\right)=M a^{i}-M \Gamma_{j k}^{i} u^{j} u^{k}=\frac{M \sigma}{m c^{3}} F^{i}-M \Gamma_{j k}^{i} u^{j} u^{k}$.

We determine the radiation flux $F^{i}$ from the expression

$F^{i}=h^{i}{ }_{j}{ }^{j k} u_{k}$, where $h_{j}^{i}=\delta^{i}{ }_{j}-u^{i} u_{j}$ is the projection tensor, and $T^{j k}$ is the stress-energy tensor for the radiation. Thus, the flux is

$F^{i}=T^{i k} u_{k}-u^{i} \epsilon$

with

$\epsilon \equiv T^{t t} u_{t} u_{t}+2 T^{t r} u_{t} u_{r}+T^{r r} u_{r} u_{r}+T^{\phi \phi} u_{\phi} u_{\phi}$.

Expressing $\varepsilon \equiv \epsilon /[\pi I(r)]$ in tetrad components, we obtain Eq. (7). We take the tetrad components of the stress-energy tensor from Abramowicz et al. (1990):

$$
\begin{aligned}
T^{(t)(t)} & =2 \pi I(r)(1-\cos \alpha) \\
T^{(t)(r)} & =\pi I(r)\left(\sin ^{2} \alpha\right) \\
T^{(r)(r)} & =\frac{2}{3} \pi I(r)\left(1-\cos ^{3} \alpha\right) \\
T^{(\phi)(\phi)} & =\frac{1}{3} \pi I(r)\left(\cos ^{3} \alpha-3 \cos \alpha+2\right),
\end{aligned}
$$

with all other components except $T^{(\theta)(\theta)}$ vanishing. The $T^{(\theta)(\theta)}$ component, when it appears, will however always be multiplied by $u^{\theta}=0$, so we may ignore this component for the present problem. In the equations above, $\alpha=\alpha(r)$ is the viewing angle and $I(r)$ is the intensity of radiation. In our dimensionless coordinates, they are given by

$$
\begin{aligned}
\sin \alpha & =\frac{R}{r} \sqrt{\frac{1-2 / r}{1-2 / R}}, \\
I(r) & =I(R)\left(\frac{1-2 / R}{1-2 / r}\right)^{2} .
\end{aligned}
$$

\section{References}

Abramowicz, M. A., Ellis, G. F. R., \& Lanza, A. 1990, ApJ, 361, 470

Bini, D., Jantzen, R. T., \& Stella, L. 2009, Class. Q. Grav., 26, 055009

Bini, D., Geralico, A., Jantzen, R. T., Semerák, O., \& Stella, L. 2011, Class. Q. Grav., 28, 035008

Damen, E., Wijers, R. A. M. J., van Paradijs, J., et al. 1990, A\&A, 237, 103

Feng, H., \& Soria, R. 2011, New Astron. Rev., 55, 166

Gladstone, J. C., Roberts, T. P., \& Done, C. 2011, Astron. Nachr., 332, 345

in 't Zand, J. J. M., Galloway, D. K., \& Ballantyne, D. R. 2011, A\&A, 525, A111 Kluźniak, W. 2013, A\&A, 551, A70

Kluźniak, W., \& Wagoner, R. V. 1985, ApJ, 297, 548

Kollmeier, J. A., Onken, C., Kochanek, C. S., et al. 2011, ApJ, 648, 128

Lewin, W. H. G., van Paradijs, J., \& Taam, R. E. 1993, Space Sci. Rev., 62, 223 Lin, D., Remillard, R. A., \& Homan, J. 2009, ApJ, 696, 1257

Oh, J. S., Kim, H., \& Lee, H. M. 2010, Phys. Rev. D, 81, 084005

Oh, J. S., Kim, H., \& Lee, H. M. 2011, New Astron., 16, 3, 183

Mishra, B., \& Kluzniak, W. 2013, A\&A, submitted

Nobuta, K., Akiyama, M., Ueda, Y., et al. 2012, ApJ, 761, 143

Phinney, E. S. 1987, in Superluminal Radio Sources, eds. J. A. Zensus, \& T. J. Pearson (Cambridge: Cambridge University Press), 12

Revnivtsev, M., Gilfanov, M., Churazov, E., \& Sunyaev, R. 2002, A\&A, 391, 1013

Stahl, A., Wielgus, M., Abramowicz, M., Kluźniak, W., \& Yu, W. 2012, A\&A, 546, A54

Walker, M. A. 1988, ApJ, 330, L47

Walker, M. A., \& Mészáros, P. 1989, ApJ, 346, 844

Wielgus, M., Stahl, A., Abramowicz, M., \& Kluźniak, W. 2012, A\&A, 545, 123 